\title{
Nondimensional Translational Characteristics of Elastomer Components
}

\section{A. Dutzler ${ }^{1,},{ }^{*}$, C. Buzzi ${ }^{1}$ and M. Leitner ${ }^{1}$}

${ }^{1}$ Institute of Structural Durability and Railway Technology, Graz University of Technology, Graz, Austria.

"corresponding author: andreas.dutzler@tugraz.at

\section{ABSTRACT}

Elastomer components are used in both primary and secondary spring stages in bogies of rail vehicles. The design of spring components of a bogie requires knowledge on the calculation of the elastic properties of these components. An elastomer spring component is typically analysed in the dimension to be investigated. Calculated force-displacement curves are directly related to the material and dimension of the component itself. The objective of this paper is to establish generalized or, in other words, universally valid force-displacement characteristics by breaking the entanglement with component size. The advantage of this approach is the extended validity of the results for a specific spring shape of any size. The simulations are performed only once for each shape and may be converted to any other size using the proposed methodology. A numerical study of a layer spring with rectangular cross-sectional area and fixed edges on both top and bottom sides serves as a reference example.

Keywords: bogie, elastomer components, spring characteristics, finite element analysis, hyperelasticity

\section{Nomenclature}

$\begin{array}{cl}A & \text { cross sectional area in } \mathrm{mm}^{2} \\ E A, G A & \text { nominal axial and shear stiffness in } \mathrm{N} \\ \overline{E A}, \overline{G A} & \text { effective axial and shear stiffness in } \mathrm{N} \\ k_{C}, k_{S} & \text { component shape factors for compression and shear loading in N/N } \\ L, B, H & \text { length, width and height in } \mathrm{mm} \\ D, d & \text { outer and inner diameter in } \mathrm{mm} \\ X, Y, Z & \text { coordinates in mm } \\ U, V, W & \text { displacements in mm } \\ F_{X}, F_{Y}, F_{Z} & \text { forces in } \mathrm{N} \\ \mu, K & \text { shear and bulk modulus (Neo-Hookean material parameters) in MPa } \\ F & \text { deformation gradient tensor } \\ J & \text { determinant of deformation gradient tensor (volume ratio) } \\ \hat{F} & \text { distortional part of the deformation gradient tensor } \\ \hat{C} & \text { distortional part of the right Cauchy Green deformation tensor } \\ \psi & \text { strain energy density per unit undeformed volume } \\ \widehat{\psi} & \text { distortional part of strain energy density per unit undeformed volume } \\ U & \text { volumetric part of strain energy density per unit undeformed volume }\end{array}$

\section{Abbreviations}

FEM finite element method

\subsection{INTRODUCTION}

Elastomer components have a wide range of application as elastic springs in bogies of rail vehicles. Due to the shape-based customizable force-deflection properties, the design of such parts is essential in order to obtain the desired mechanical behaviour. For simple geometries, i.e. a rubber block bonded between two parallel steel plates, it is shown that the elastic modulus in compression depends on the shape [1]. Several formulas guided by experiments have been proposed [1-2]. However, in numerical simulations of elastomer components and their mostly nonlinear spring characteristics, the finite element method is widely used nowadays [3-6]. For this purpose, the component is typically analysed in the dimension to be investigated. The term dimension in this context means the component size, for example the length, width and height of a component. Using an incremental approach to 
solve the equilibrium states according to stepwise externally applied displacements (deflections) or forces, the relationship displacement vs. force is calculated. This simulation result at the boundary condition is dimensionally dependent and directly associated with the component size.

The objective of this paper is to establish generalized force-displacement characteristics by breaking the entanglement with component size. The structure of the paper is as follows; first, the geometry including kinematics and the load cases of the simulation model is defined. In the second step, the FEM model with the associated evaluation methodology is presented, followed by the methodology for creating nondimensional characteristic curves. Finally, a numerical example of a layer spring with rectangular cross-section and bonded on both top and bottom faces is shown. The presented methodology enables to scale spring characteristics to various component sizes and materials.

\subsection{SIMULATION MODEL DESCRIPTION}

An elastic structural component contains two connection points as interface to its environment, at which displacements and forces are transmitted (see Fig. 1). The connection point 1 at the coordinate origin is fixed without restriction of validity. External forces are applied at connection point 2, where displacements are also evaluated. As elastomers exhibit nearly incompressible material behaviour, appropriate simulation techniques are applied in FEM analyses to overcome the so-called volumetric locking effect. Common practice is to use eightnode hexahedral elements with linear shape functions for the interpolation of displacements in combination with the element-wise constant fields of hydrostatic pressure and volume change [7-8]. This technique is called selective three-field variation or $(u, p, J)$ - formulation. Isotropic hyperelastic material behaviour is assumed for the description of rubber, i.e. the mechanical behaviour of rubber is idealized as a pure (isotropic) elastic material. Viscoelastic and -plastic effects are neglected. In the framework of hyperelasticity, the stress-strain relation is derived from a potential and hence, the deformation due to externally applied loads is independent of the load sequence. The isotropic hyperelastic Neo-Hookean material formulation is chosen as the constitutive law due to its simplicity and good agreement with experiments on natural rubber samples [9]. In a nearly incompressible isotropic hyperelastic material formulation, the strain energy density function per unit undeformed volume is additively composed of contributions from a multiplicative decomposition of the deformation gradient tensor into a distortional (volume-constant) and a dilatational (volume-changing) part [11] (see Equations 1-4). The NeoHookean material model is finally applied only on the distortional part of the deformation whereas the uncoupled bulk behaviour is modeled by a penalty term, both shown in Equation (5).

$$
\begin{aligned}
& F=1+\frac{\partial u}{\partial X} \\
& \hat{F}=J^{-1 / 3} F \text { and } J=\operatorname{det}(F) \\
& \hat{C}=\hat{F}^{\mathrm{T}} \hat{F} \\
& \psi=\hat{\psi}(\hat{C})+U(J) \\
& \hat{\psi}=\frac{\mu}{2}(\operatorname{tr}(\hat{C})-3) \text { and } U(J)=\frac{K}{2}(J-1)^{2}
\end{aligned}
$$
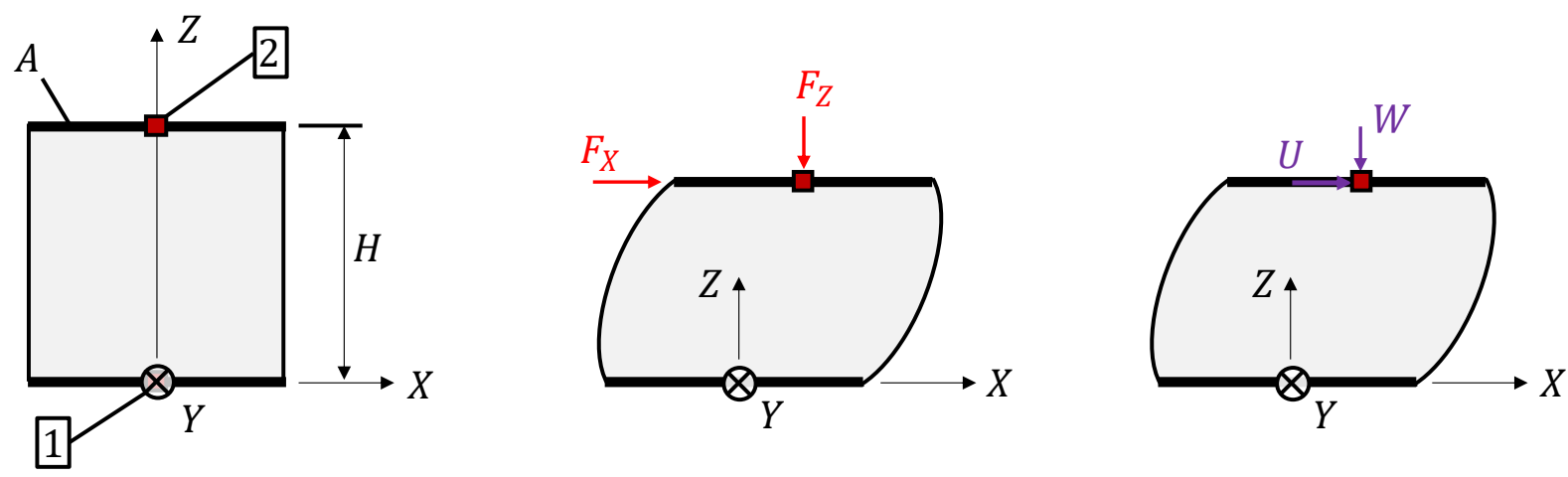

Figure 1. Kinematics and kinetics of an elastomer spring with cross section A and connection points 1 and 2 in the undeformed state (left), and internal forces and displacements in the finite deformed state (center, right) 


\subsection{LOAD CASE DEFINITION}

Elastomer components usually have an axis along their primarily used direction. For modeling purposes, a nonlinear force-displacement characteristic is assumed along this axis. This axis is referred to as the primary axis in this paper. The two axes perpendicular to the primary axis are designated as secondary axes. A linear forcedisplacement behaviour is assumed along the secondary axes and the translational stiffnesses are determined as a function of the primary state of deflection. However, no influence of the secondary deflection state on the primary spring characteristic is considered. In the primary axial load case, the normal compressive force is evaluated as a function of the primary axial spring deflection (see Fig. 2 left). In the secondary shear load case the shear stiffness is evaluated as a function of the primary axial spring deflection (see Fig. 2 right). Reaction (bending) moments due to the shear deflection are neglected as this paper focuses only on translational component curves.
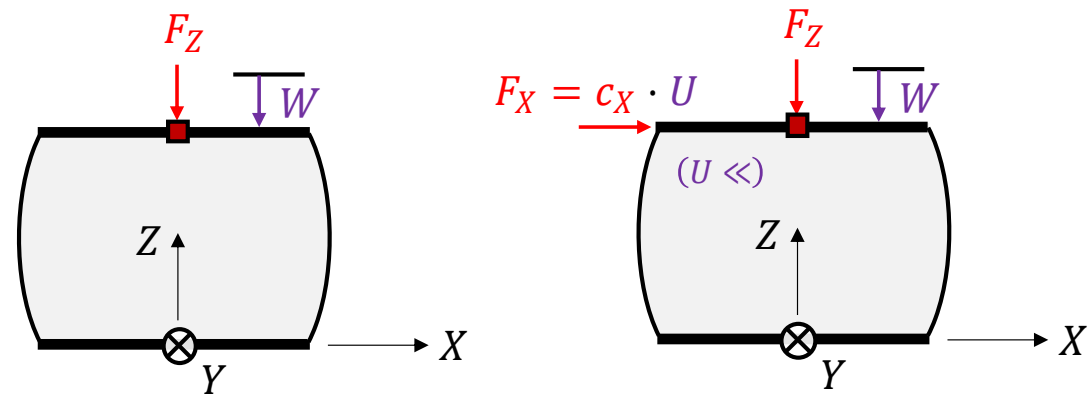

Figure 2. Primary compression (left) and secondary linearized shear load case of an elastomeric spring (right)

\subsection{DETERMINATION OF NOMINAL STIFFNESS VALUES}

Following the theory of bars and beams, the product of cross-sectional area and modulus of elasticity is referred to as axial (compressive) stiffness $E A$, while the product of cross-sectional area and shear modulus is shear stiffness $G A$. The material parameters modulus of elasticity and shear modulus are determined by material tests. If the material characteristic curve is nonlinear, as common for elastomers, these material parameters must be those for the undeformed state as for the uniaxial force-stretch behaviour given in Equation (6). Formulas for the calculation of exemplaric cross sections of rectangular shape as well as circular ring cross sections are given in Equation (7).

$$
\begin{aligned}
& E=\lim _{l / L \rightarrow 1} \frac{d\left(F_{\text {uniaxial }} / A\right)}{d(l / L)} \\
& A_{\square}=L B \quad \text { and } \quad A_{\odot}=\frac{\left(D^{2}-d^{2}\right) \pi}{4}
\end{aligned}
$$

\subsection{CALCULATION OF COMPONENT SHAPE FACTORS}

The component shape factor is the stiffness determined on the component behaviour in relation to the corresponding nominal stiffness. In a component axial force-displacement curve, the primary axial stiffness at some primary axial state of deflection is determined as the slope of the curve. Multiplied by the component height, this leads to the state of deformation - dependent effective component stiffness as the "effective" product $\overline{E A}$ of elastic modulus and area as shown in Equation (8). These effective component related values are denoted with an overbar to distinguish them from the nominal stiffness values. The component shape factors for compression and shear are defined as the ratio of effective component stiffness and nominal stiffness as given in Equation (9).

$$
\begin{array}{lll}
\overline{E A}=H \cdot \frac{d F_{Z}}{d W} & \text { and } & \overline{G A}=H \cdot \frac{d F_{X}}{d U} \\
k_{C}=\frac{\overline{E A}}{E A} & \text { and } & k_{S}=\frac{\overline{G A}}{G A}
\end{array}
$$

\subsection{NUMERIC EXAMPLE: NONDIMENSIONAL TRANSLATIONAL CHARACTERISTICS OF A LAYER SPRING}

According to Fig. 3 (left), consider a layer spring with rectangle cross-sectional shape and dimensions of $B=$ $200 \mathrm{~mm}, L=100 \mathrm{~mm}$ and $H=25 \mathrm{~mm}$ with an elastomer shear modulus $\mu=0.6 \mathrm{MPa}$ (incompressible). We are looking for; a) force-displacement curves for compression loading with $W=0 \ldots-5 \mathrm{~mm}$, and combined 
compression and shear loading with $W=0 \ldots-5 \mathrm{~mm} ; U=0 \ldots 10 \mathrm{~mm}$ as well as; b) component shape factors for compression and shear as a function of the primary deflection $(W / H)$.

\subsection{Simulation model}

The mesh of the FEM simulation model consists of eight-noded hexahedral elements as described in section 2. These elements are uniformly distributed over the volume and have minimum and maximum edge lengths of $2.1 \mathrm{~mm}$ and $3.3 \mathrm{~mm}$, respectively. The model includes 24,483 nodes, 21,600 elements and 73,749 displacement degrees of freedom as well as 21,600 compression and 21,600 volume change degrees of freedom as shown in Figs. 3 and 4. Due to the required incompressible material behaviour, the bulk modulus is chosen 5,000 times larger than the shear modulus [9]. The nominal stiffness values are determined as $E A=36,000 \mathrm{~N}$ and $G A=12,000$ N. Simulations are performed with both MSC.Marc Mentat 2020 and inhouse FEM code written in Python which lead to identical results. ParaView 5.9 is used for the visualization of the deformed model. Simulation results are overall displacements of the elastomer and both forces and displacements on the top plate.
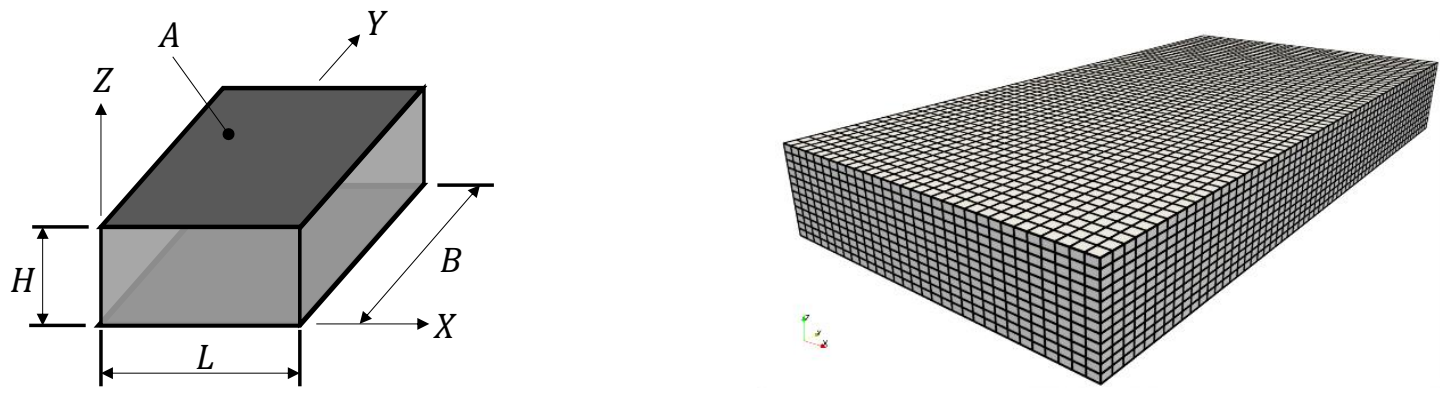

Figure 3. Sketch (left) and FEM model (right) of a rectangular layer spring with rigid boundaries at top and bottom. FEM model with $(30,60,12)$ - hexahedral elements along the $(X, Y, Z)$ axes
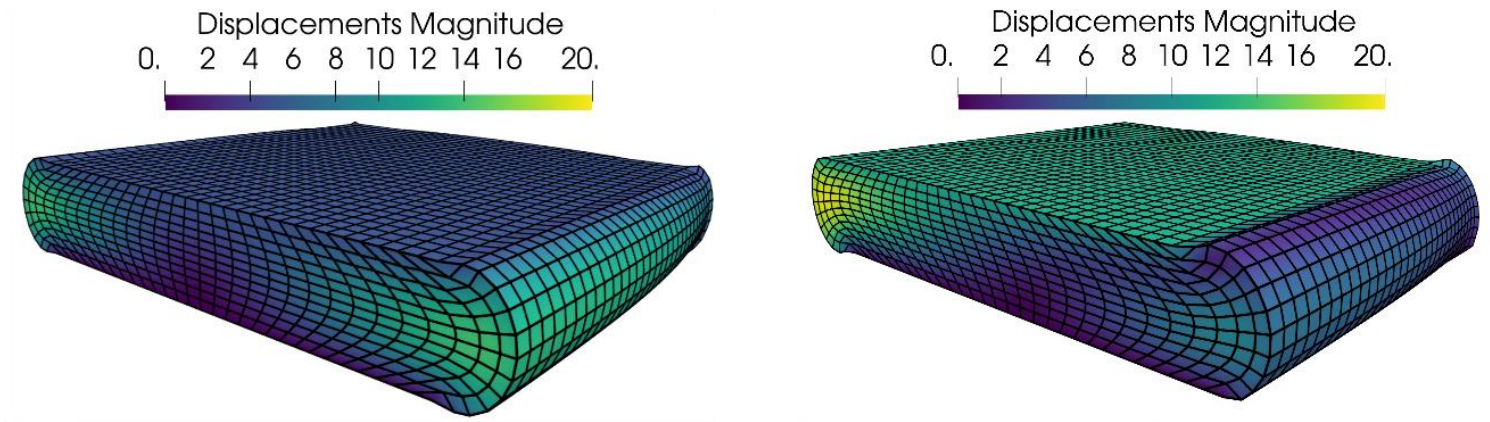

Figure 4. FEM simulation result: half-model with symmetry plane XZ (contour plot of displacements magnitude). Primary compression loading $\mathrm{W}=-5 \mathrm{~mm}$ (left) and combined compression and shear loading $\mathrm{W}=-$ $5 \mathrm{~mm}$ and $\mathrm{U}=10 \mathrm{~mm}$ (right)

\subsection{Component characteristics}

The primary axial compressive force-displacement curve is shown in Fig. 5 (left) where both negative signs of force and displacement data have been dropped. The secondary shear force-displacement curve for a combined compression and shear loading along the short side of the component is shown in Fig. 5 (right). Nondimensional plots are shown in Fig. 6 for both compression and combined compression and shear loadings. In all plots from Figs. 5 and 6, FEM results are denoted as dots whereas the continuous lines are obtained with a spline interpolation of the discrete FEM results. The dashed line in Fig. 6 (left) represents the linearized solution of uniaxial compression in the undeformed state, or with other words, the linearized solution of a rubber block with idealized lubricated instead of bonded top and bottom plates. 

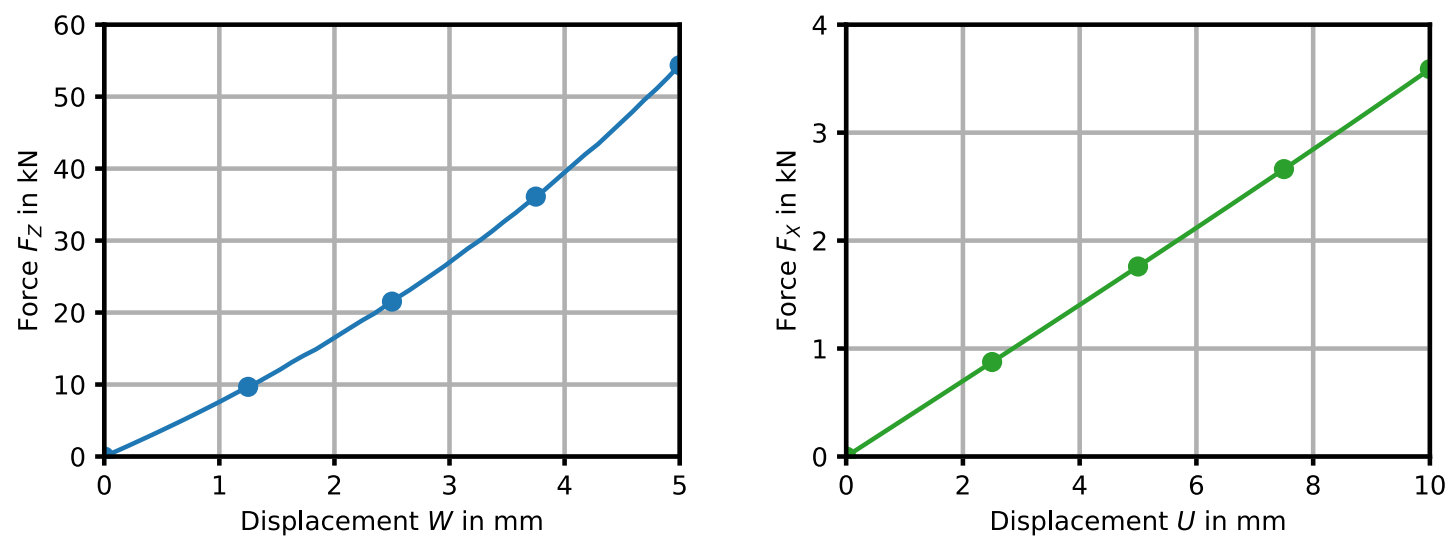

Figure 5. Force-displacement curves of primary compression loading (left) and combined compression and shear loading $\mathrm{W}=-5 \mathrm{~mm}$ and $\mathrm{U}=10 \mathrm{~mm}$ (right)
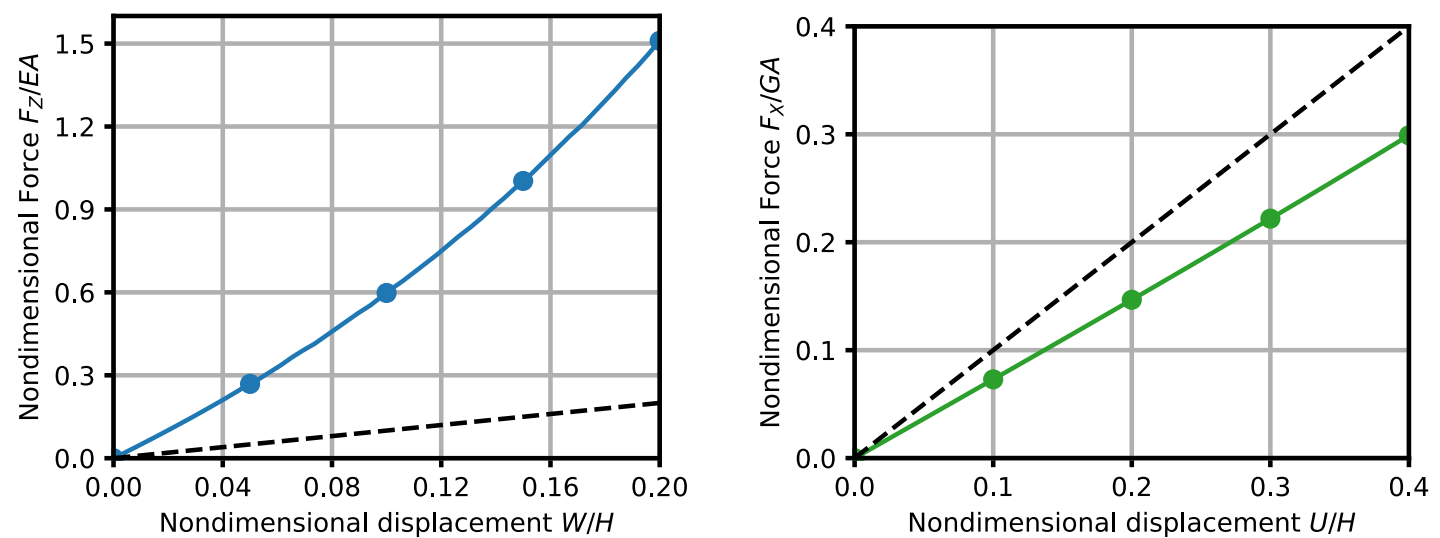

Figure 6. Nondimensional force-displacement curves of primary compression loading (left) and combined compression and shear loading $\mathrm{W}=-5 \mathrm{~mm}$ and $\mathrm{U}=10 \mathrm{~mm}$ (right). A dashed line represents a slope of one

\subsection{Component shape factors}

The component shape factors for compression and shear loading as a function of the primary deflection are shown in Fig. 7. These curves are obtained as the slope of the curves shown in Figs. 5 and 6. The component shape factor for compression evaluates to $\overline{E A} / E A=4.9$ whereas the component shape factors for shear evaluate to $\overline{G A}_{X} / G A=0.95$ (short side) and $\overline{G A}_{Y} / G A=0.97$ (long side) in the undeformed state. 

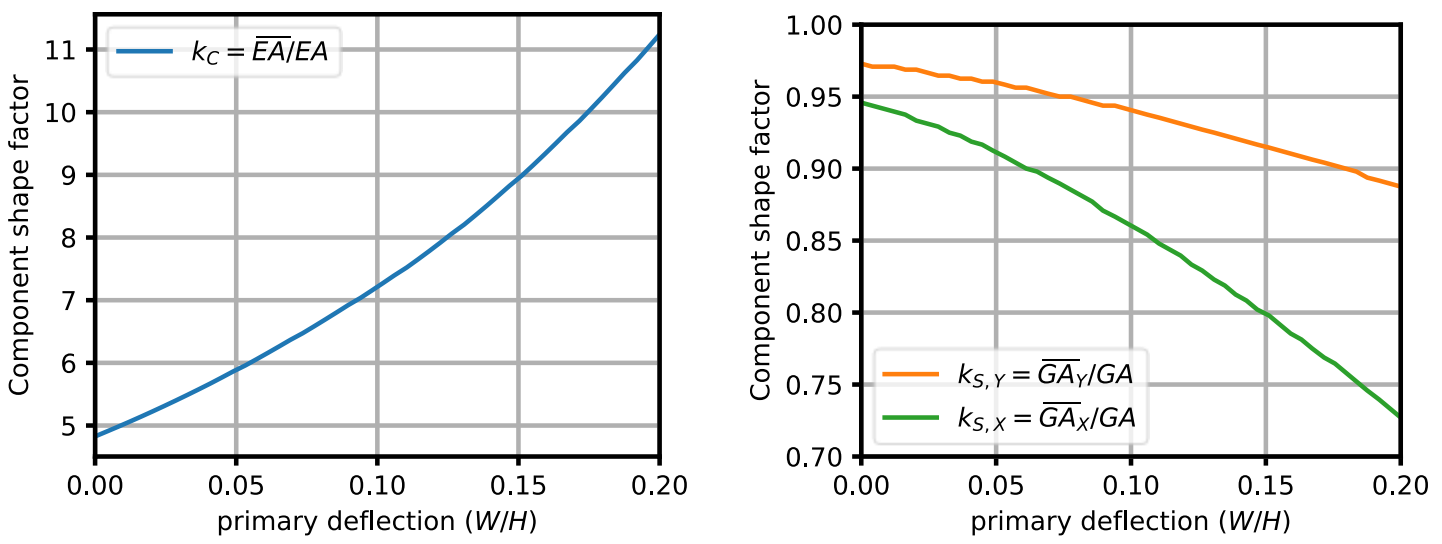

Figure 7. Component shape factors versus primary compression loading for compression (left) and shear loading (right)

\subsection{Discussion of results}

In Fig. 7 (left), it is clearly visible that the layer spring exhibits progressive increasing axial stiffness with raising axial deflection. In compression loading, the initial component shape factor is nearly five which may be interpreted in several ways; either the effective area is nearly five times bigger than the geometrical cross-sectional area, or the effective modulus of elasticity is five times bigger than the modulus determined in the material characterization. Another possibility is the interpretation as effective vs. nominal stiffness ratio which makes no assumption of the change in elastic moduli or area. This seems to be the most general way to interpret these results and therefore the last-mentioned definition is preferred. An empirical formula for square prisms as denoted in [1] gives exactly the same result for the undeformed state $(W / H=0)$. The calculated component shape factors for compression loading is also within the range of values evaluated by experiments $(\overline{E A} / E A=4.1$ to 5.5$)$ found in textbooks [12-13].

For the case of shear loading, the component shape factor starts closely at one and decreases with raising primary deflection. This behaviour is well explained in literature [14]; first, the height of the rubber block decreases while the cross-sectional area of the rubber increases due to incompressiblity-induced bulging in compression loading. Lastly, the applied compressive force contributes to the total shear force. Although both component shape factors in shear loading decrease with increasing axial deflection, note that the factor associated to the short side of the part exhibits a higher decrease than the one associated to the long side of the layer spring (see Fig. 7 right). This is mainly due to the bulging behaviour of the rubber block. A higher amout of bulging is observed in the XZ plane containing the smaller cross-sectional area (see Fig. 4) compared to the YZ plane with higher cross-sectional area.

\subsection{CONCLUSION}

With the proposed methodology, it is enabled to detach simulated translational spring characteristics with nonlinear behaviour along the primary axis from their dimension. This is mainly used to create characteristic diagrams of a specific shape of spring, which again serve as a basis for the calculation of a dimension of interest. The advantage of this approach is the extended validity of the results for a specific spring shape of any size. The simulations are performed only once for each shape and can be converted to any size using the presented methodology. In the future, the presented methodology will be extended to rotational characteristics in order to cover the entire spring behaviour. By varying the geometry parameters, the influence of the shape design can also be incorporated into the representation of the component shape factors from Fig. 7 in which a complete characteristics diagram of a spring type is obtained. Once these diagrams have been established for several spring types, the selection of a suitable elastomer component is a simple task in the design of spring components in bogies of rail vehicles.

\section{REFERENCES}

[1] A. N. Gent and P. B. Lindley, "The Compression of Bonded Rubber Blocks", Proceedings of the Institution of Mechanical Engineers, vol. 173, no. 1, pp. 111-122, Jun. 1959.

[2] L. S. Porter and E. A. Meinecke, "Influence of Compression upon the Shear Properties of Bonded Rubber Blocks”, Rubber Chemistry and Technology, vol. 53, no. 5, pp. 1133-1144, Nov. 1980. 
[3] D. W. Nicholson, N. W. Nelson, B. Lin, and A. Farinella, "Finite Element Analysis of Hyperelastic Components", Applied Mechanics Reviews, vol. 51, no. 5, pp. 303-320, May 1998.

[4] D. F. Lalo, M. Greco, and M. Meroniuc, "Numerical Modeling and Experimental Characterization of Elastomeric Pads Bonded in a Conical Spring under Multiaxial Loads and Pre-Compression", Mathematical Problems in Engineering, vol. 2019, pp. 1-14, Feb. 2019.

[5] R. K. Luo, L. M. Peng, X. P. Wu, and W. J. Mortel, "Load-deflection prediction and stress verification in extreme large deformation of rubber suspensions used in rail vehicles", Proceedings of the Institution of Mechanical Engineers, Part F: Journal of Rail and Rapid Transit, vol. 226, no. 2, pp. 228-232, Aug. 2011.

[6] P. Wriggers, Nonlinear finite element methods. Springer Berlin, 2010.

[7] J. Bonet, A. J. Gil, and R. D. Wood, Nonlinear solid mechanics for finite element analysis: Statics, Cambridge University Press, 2016.

[8] J. C. Simo, R. L. Taylor, and K. S. Pister, "Variational and projection methods for the volume constraint in finite deformation elasto-plasticity", Computer Methods in Applied Mechanics and Engineering, vol. 51, no. 1-3, pp. 177-208, 1985.

[9] G. Marckmann and E. Verron, "Comparison of Hyperelastic Models for Rubber-Like Materials", Rubber Chemistry and Technology, vol. 79, no. 5, pp. 835-858, Nov. 2006.

[10] R. W. Ogden, "Elastic Deformations of Rubberlike Solids" in Mechanics of Solids, Elsevier, 1982, pp. 499-537.

[11] P. J. Flory, "Thermodynamic relations for high elastic materials", Transactions of the Faraday Society, 1985, vol. 57, pp. 829-838.

[12] E. F. Göbel, Gummifedern. Berechnung und Gestaltung, Springer Berlin Heidelberg, 1969.

[13] W. Battermann and R. Köhler, "Elastomere Federung. Elastische Lagerung", Wilhelm Ernst und Sohn Berlin München, 1982.

[14] L. S. Porter and E. A. Meinecke, "Influence of Compression upon the Shear Properties of Bonded Rubber Blocks", Rubber Chemistry and Technology, vol. 53, no. 5, pp. 1133-1144, Nov. 1980. 